Nephrologe 2011 · 6:389-389

DOI 10.1007/s11560-011-0561-3

(c) Springer-Verlag 2011

\section{G. Wolf ${ }^{1} \cdot$ O. Witzke ${ }^{2}$}

${ }^{1}$ Klinik für Innere Medizin III, Universitätsklinikum Jena

${ }^{2}$ Klinik für Nephrologie, Universitätsklinikum Essen, Universität Duisburg-Essen

\title{
Nieren- und Diabeteserkrankungen
}

Die diabetische Nephropathie ist weiterhin die Erkrankung, die in Deutschland am häufigsten zur dialysepflichtigen Niereninsuffizienz führt. Dieses Krankheitsbild wurde zuletzt umfassend im September 2007 in Der Nephrologe abgehandelt. Rüster und Wolf beschreiben neue Aspekte zur Pathogenese der diabetischen Nephropathie. Die sich daraus ergebenden neuen Therapiestrategien, wie eine Vitamin-D-Rezeptor-Aktivierung durch Vitamin-D-Analoga, müssen allerdings erst in größeren Studien geprüft werden.

Weiterhin entscheidend für den Krankheitsverlauf und die Verminderung der Progression einer diabetischen Nephropathie ist eine gute Stoffwechseleinstellung. Hasslacher, der das Thema schon im Jahr 2007 in Der Nephrologe beschrieben hatte, erwähnt nun in seinem Übersichtsartikel die Probleme der antidiabetischen Therapie bei Niereninsuffizienz und geht auch auf neuere Medikamente wie $\mathrm{DPP}_{4}$ Inhibitoren und GLP-Analoga ein, die 2007 noch nicht auf dem Markt waren.

Wolfgang Pommer beschreibt die unterschiedlichen Möglichkeiten der Nierenersatztherapie beim Diabetes mellitus und erwähnt insbesondere, dass die Peritonealdialyse in Deutschland leider viel zu wenig zum Einsatz kommt.

Die Autoren dieser Ausgabe gehen aber über eine Beschreibung der reinen diabetischen Nephropathie hinaus. Helmut Arbogast aus München beschreibt die Indikation und die Ergebnisse der Nieren-Pankreas-Transplantation, dem Standardverfahren zur Behandlung von Typ-1-Diabetikern mit terminaler Niereninsuffizienz, das aber auch in ausgewähl- ten Fällen bei Typ-2-Diabetes-mellitus eingesetzt werden kann.

Leider ist ein neu aufgetretener Diabetes mellitus eine häufige Komplikation nach Transplantation und beeinflusst das Patienten- und Transplantatüberleben negativ. Hierbei spielen natürlich die unterschiedlichen immunsuppressiven Medikamente (Kortikosteroide und Calcineurininhibitoren), aber auch Virusinfekte eine wichtige Rolle. Türk und Witzke beschreiben die Pathogenese und mögliche Therapiestrategien bei diesem häufigen Krankheitsbild in der Transplantationsmedizin.

Nieren- und Diabeteserkrankungen sind weiterhin ein großes klinisches Problem und erfordern eine enge interdisziplinäre Zusammenarbeit von Nephrologen, Diabetologen, Transplantationsmedizinern, Hausärzten und Internisten. Wir hoffen, dass wir mit dem vorliegenden Heft das Interesse des klinisch tätigen Nephrologen an den wichtigen Erkrankungen im Schnittpunkt zwischen Diabetologie und Nephrologie geweckt haben.

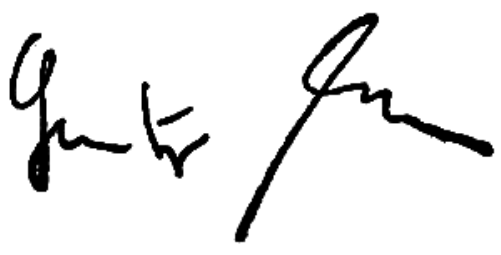

Prof. Dr. med. Gunter Wolf, Jena

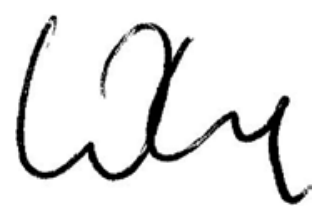

Prof. Dr. med. Oliver Witzke, Essen

\section{Korrespondenzadressen \\ Prof. Dr. G. Wolf}

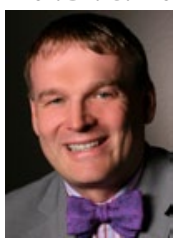

Klinik für Innere Medizin III, Universitätsklinikum Jena 07740 Jena gunter.wolf@med.uni-jena.de

\section{Prof. Dr. O. Witzke}

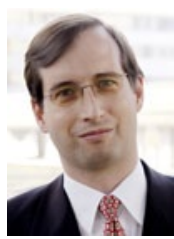

Klinik für Nephrologie, Universitätsklinikum Essen, Universität Duisburg-Essen Hufelandstrasse 55, 45122 Essen oliver.witzke@uk-essen.de 